\title{
Cryosectioning of Cultured Cells on Permeable Support
}

\author{
Yuki Tajika', Toshiyuki Matsuzaki', Takeshi Suzuki', Takeo Aoki', \\ Haruo Hagiwara ${ }^{1}$ and Kuniaki Takata ${ }^{1}$ \\ ${ }^{1}$ Department of Anatomy and Cell Biology, Gunma University School of Medicine, Maebashi, Gunma 371-8511, Japan
}

Received March 3, 2003; accepted March 13, 2003

\begin{abstract}
Immunolabeling of cultured cell lines of epithelial origin is often performed to study cell polarity. To explore the localization of cellular molecules in detail, we devised an efficient and convenient method to prepare semithin and ultrathin cryosections of cells cultured on permeable support. Multiple specimens were piled up to obtain many vertical images of cells in a single section. Freezing
\end{abstract}

\begin{abstract}
them in cooled $n$-hexane resulted in clear and homogeneous frozen specimens, from which semithin and ultrathin cryosections of epitherial cells on permeable support were easily obtained. With this method, it is possible to prepare many cryosections of good quality for histochemical examination of vertical images of cells.
\end{abstract}

Key words: cryosectioning, MDCK cells, permeable support

Sectioning of frozen specimens and retrieval of sections with a small sucrose droplet was invented by Tokuyasu [7]. Since then, cryomicrotomy has been greatly improved [4]. Immunohistochemical labeling on semithin and ultrathin cryosections is widely used to study localization of cellular molecules. Polarized epithelial cells contain distinct apical and basolateral plasma membrane domains and underlying cytoplasmic structure. For studies on epithelial cell polarity, cultured cell lines of epithelial origin have been used. To examine whether molecules of interest are in the apical or basolateral region, vertical sectioning images of cultured epithelial cells are often generated from consecutive optical sections obtained by a confocal microscope. However, the quality of $\mathrm{X}-\mathrm{Z}$ or $\mathrm{Y}-\mathrm{Z}$ images is not satisfactory partly due to low mechanical precision along the Z-axis and/or possible artifacts accumulated during the digital reconstruction process. Semithin cryosections can provide extremely clear images of vertical sections containing detailed intracellular information [5]. Here we show a convenient procedure for the preparation of semithin cryosections of epithelial cells cultured on permeable support.

Correspondence to: Kuniaki Takata, Department of Anatomy and Cell Biology, Gunma University School of Medicine, 3-39-22 Showamachi, Maebashi, Gunma 371-8511, Japan.
Madin-Darby Canine Kidney (MDCK) cells [1, 2] were fed on permeable support $\left(8 \times 10^{4}\right.$ cells/well, Transwell cell culture insert \#3401, Corning Coaster, Cambridge, MA, USA), and were incubated at $37^{\circ} \mathrm{C}$ in an atmosphere of $5 \%$ $\mathrm{CO}_{2}$ air. Transepithelial electrical resistance (TER) value was measured with a Millicell-ERS (Millipore, Billerica, MA, USA) to evaluate the level of confluence. Cells were incubated untill the TER value rose above $2000 \Omega \cdot \mathrm{cm}^{2}$, fixed with $3 \%$ paraformaldehyde in $0.1 \mathrm{M}$ sodium phosphate buffer, pH 7.4 (PB) for $20 \mathrm{~min}$, and infiltrated with $2.3 \mathrm{M}$ sucrose in $\mathrm{PB}$ over night. The permeable support was separated from the insert, cut into small square pieces, and piled up on the L-shaped specimen carrier (Fig. 1). It was immersed and frozen in an appropriate cryogen placed in cryokit chamber at $-75 \sim-65^{\circ} \mathrm{C}$.

We tested freezing in liquid nitrogen $\left(\mathrm{LN}_{2}\right)$, cooled $\mathrm{n}$-hexane, and isopentane. When specimens were frozen by direct immersion in $\mathrm{LN}_{2}$, many bubbles formed around the specimens. This may prevent the rapid conduction of heat due to formation of barrier layer of nitrogen gas around specimens. The surface of the specimen was opaque, and obtaining good sections from them was difficult. Separation of the cell monolayer from the permeable support was often encountered. To avoid such adverse phenomena, specimens can be frozen in an appropriate cryogen. Good cryogens 


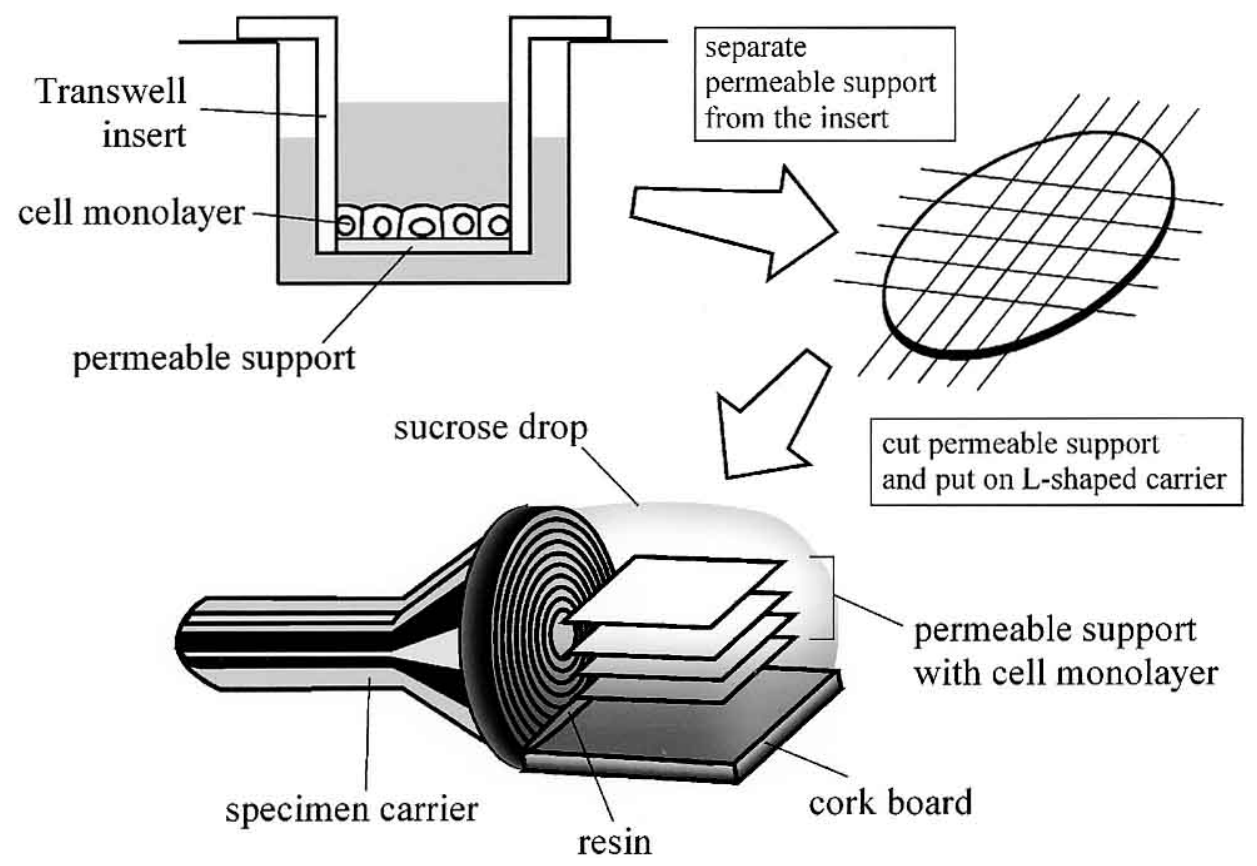

Fig. 1. Preparation of cell monolayer block using L-shaped specimen carrier. Small pieces of the cell monolayer on permeable support are piled on a thin slice of cork board attached to the specimen carrier by epoxy resin. The cork is soft enough to be trimmed off by metal blade at low temperature, and can be easily removed before sectioning. Use of OCT compound instead of epoxy resin enables the carrier to be recycled, though it is not so strong as epoxy resin.

Table 1. Commonly used cryogens

\begin{tabular}{lcc}
\hline cryogen & $\begin{array}{c}\text { boiling point } \\
\left({ }^{\circ} \mathrm{C}\right)\end{array}$ & $\begin{array}{c}\text { melting point } \\
\left({ }^{\circ} \mathrm{C}\right)\end{array}$ \\
\hline n-hexane & 69 & -95 \\
isopentane & 27 & -160 \\
propane & -42 & -188 \\
chlorodifluoromethane (Freon 22)* & -40 & -159 \\
nitrogen & -196 & -210 \\
\hline
\end{tabular}

Values are from the Chemical WebBook (http://webbook.nist.gov/ chemistry/) by the National Institute of Standards and Technology (USA). * Freon is not available because of its adverse effect on the ozone layer.

should have high boiling points and low freezing points (Table 1). Freezing with n-hexane or isopentane as a cryogen resulted in clear and homogeneously frozen specimens. No cracks were formed during sectioning. n-Hexane was convenient as a cryogen whose melting point is low enough to freeze and cut specimens in the cryokit chamber [3]. In addition, its high boiling point enables it to be stored easily at room temperature. Isopentane (2-methylbutane) is also useful, but its rubbery form near the melting point is not easy to handle.

For cryosectioning, specimens were set on Leica Ultracut S UCT ultramicrotome (Vienna, Austria) equipped with a FCS cryokit, and a slice of cork was trimmed off with a metal blade. Semithin cryosections of $0.5-1 \mu \mathrm{m}$ thick were cut. The sections were picked up with retrieval solution consisting of $1 \%$ methylcellulose- $1.15 \mathrm{M}$ sucrose, and placed on
poly-L-lysine-coated glass slides. Nomarski-differential interference images of sections showed that attachment of MDCK cells to permeable support was successfully maintained (Fig. 2A). Vertical sections of many cell layers were easily obtained.

Figure 2B shows a section of MDCK cells immunolabeled for a tight junction protein ZO-1. Sections were incubated with rat anti-ZO-1 antibody (1:500; Chemicon International, Temecula, CA, USA), and lissamine rhodamine sulfonyl chloride (LRSC)-conjugated donkey anti-rat IgG antibody (1:500; Jackson Immunoresearch, West Grove, PA, USA) as described previously [6]. The localization of ZO-1 (tight junction) was clearly seen in the cell-cell contact region at the apical side. Compared with the reconstructed vertical sectioning image by confocal microscopy, semithin cryosections provided detailed and precise localization of the antigen. Overlay of fluorescence images with Nomarski images further helped to determine the accurate localization of the label. After further trimming, ultrathin cryosections could be prepared from the same specimen and examined with a JEOL JEM-100CXII electron microscope (Tokyo, Japan). As shown in Figure 3, vertical images of epithelial cells on permeable support was easily obtained.

In conclusion, an efficient and convenient method has been devised to prepare vertical semithin and ultrathin cryosections of cells cultured on permeable support. This method allows us to perform immunolabeling using as many kinds of antibodies as the number of sections prepared from the same block. 

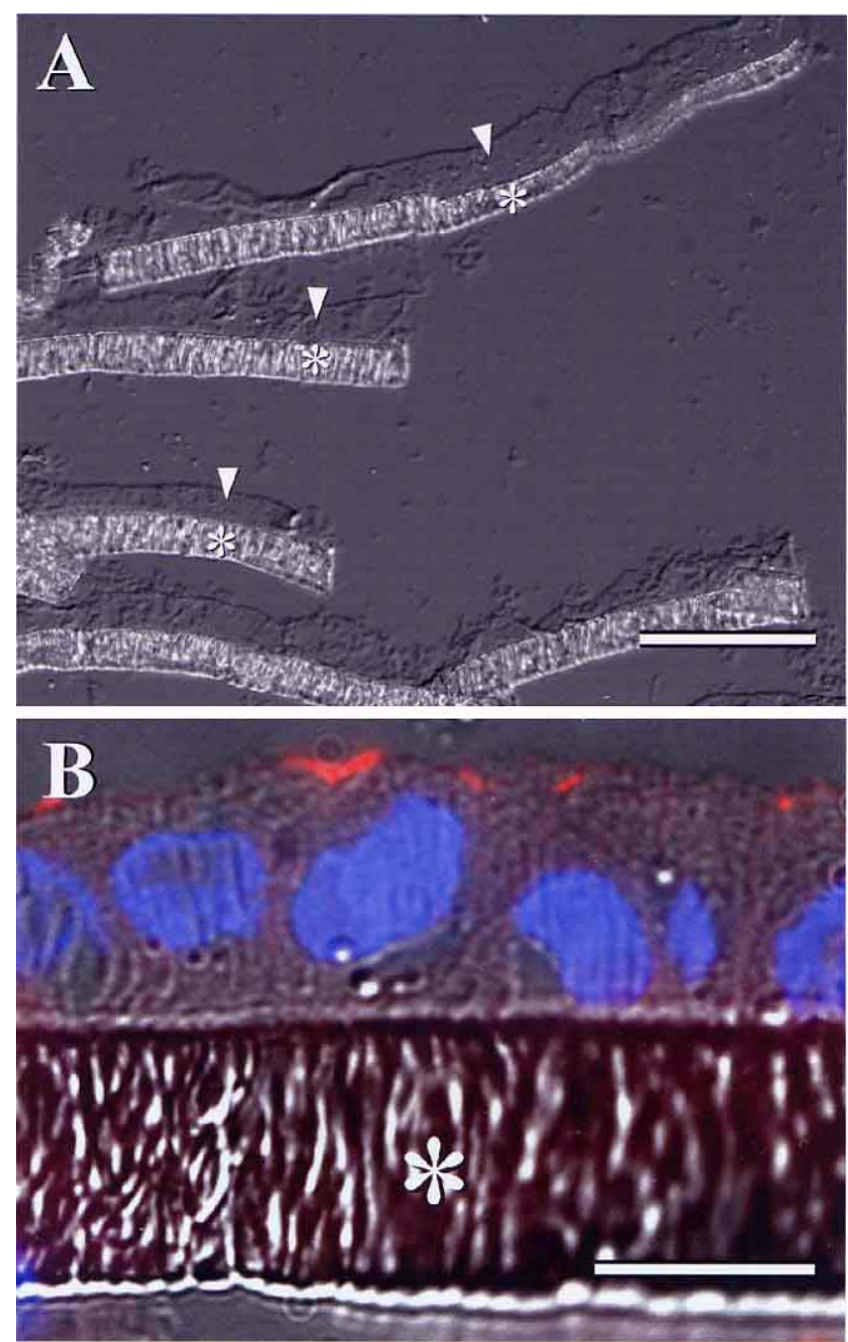

Fig. 2. (A) Semithin cryosection of $1 \mu \mathrm{m}$ thickness. Cell monolayers of MDCK cells (arrowheads) and permeable supports $(*)$ are seen. Bar=50 $\mu \mathrm{m}$. (B) Immunofluorescence staining. The localization of ZO-1 is shown in red. Nuclear DNA is stained with DAPI (blue). The permeable support is indicated by asterisk. Overlay of fluorescence image on Nomarski image. Bar $=10 \mu \mathrm{m}$.
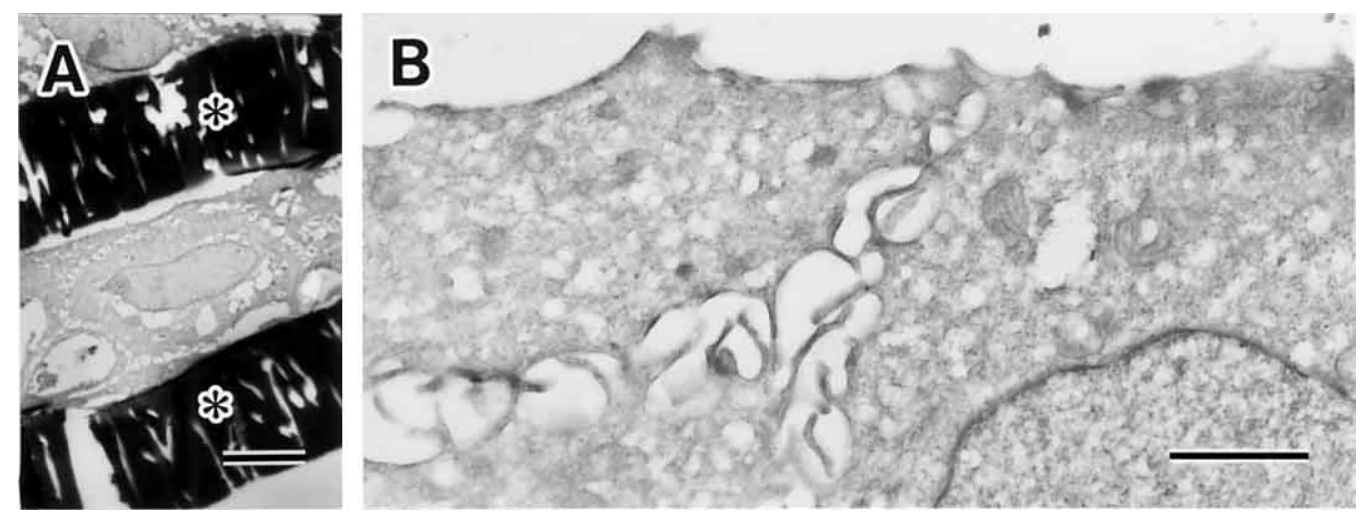

Fig. 3. Electron micrographs of MDCK cells on permeable support. Utrathin cryosections were embedded in $0.2 \%$ uranyl acetate- $2 \%$ methylcellulose. (A) A survey view. MDCK cells on permeable support $\left({ }^{*}\right)$ are seen. Bar $=5 \mu \mathrm{m}$. (B) Enlargement of the apical region of a monolayer. Bar $=1 \mu \mathrm{m}$. 


\section{Acknowledgments}

We wish to thank Ms. F. Miyata and Ms. Y. Takahashi for secretarial and technical assistance. This work was supported in part by Grants-in-Aid for Scientific Research from the Ministry of Education, Culture, Sports, Science and Technology of Japan.

\section{References}

1. Braun, A., Hammerle, S., Suda, K., Rothen-Rutishauser, B., Gunthert, M., Kramer, S. D. and Wunderli-Allenspach, H. (2000) Cell cultures as tools in biopharmacy. Eur. J. Pharm. Sci. (Suppl.) 2; S51-60.

2. Brown, D. and Breton, S. (2000) Sorting proteins to their target membranes. Kidney Int. 57; 816-824.
3. Kawamoto, T. and Shimizu, M. (2000) A method for preparing 2to 50-micron-thick fresh-frozen sections of large samples and undecalcified hard tissues. Histochem. Cell Biol. 113; 331-339.

4. Liou, W., Geuze, H. J. and Slot, J. W. (1996) Improving structural integrity of cryosections for immunogold labeling. Histochem. Cell Biol. 106; 41-58.

5. Suzuki, T., Fujikura, K., Koyama, H., Matsuzaki, T., Takahashi, Y. and Takata, K. (2001) The apical localization of SGLT1 glucose transporter is determined by the short amino acid sequence in its N-terminal domain. Eur. J. Cell Biol. 80; 765-774.

6. Tajika, Y., Matsuzaki, T., Suzuki, T., Aoki, T., Hagiwara, H., Tanaka, S., Kominami, E. and Takata, K. (2002) Immunohistochemical characterization of the intracellular pool of water channel aquaporin-2 in the rat kidney. Anat. Sci. Int. 77; 189-195.

7. Tokuyasu, K. T. (1973) A technique for ultracryotomy of cell suspensions and tissues. J. Cell Biol. 57; 551-565. 